\title{
UA promotes epithelial-mesenchymal transition in peritoneal mesothelial cells
}

\author{
CHAO-YANG DUAN ${ }^{1}$, JIN HAN $^{1}$, CHONG-YU ZHANG ${ }^{2}$, KUNYI WU $^{3}$ and YAN LIN ${ }^{4}$ \\ ${ }^{1}$ Department of Nephrology, ${ }^{2}$ National and Local Joint Engineering Research Center of Biodiagnostics and Biotherapy, \\ and ${ }^{3}$ Core Research Laboratory, The Second Affiliated Hospital of Xi'an Jiaotong University, Xi'an, Shaanxi 710004; \\ ${ }^{4}$ Department of Endocrine and Metabolic Diseases, Shaanxi Provincial People's Hospital, Xi'an, Shaanxi 710068, P.R. China
}

Received May 25, 2018; Accepted December 6, 2018

DOI: $10.3892 / \mathrm{mmr} .2019 .10476$

\begin{abstract}
Long-term peritoneal dialysis is often limited or interrupted due to the development and progression of peritoneal fibrosis. Accumulating evidence suggests that epithelial-mesenchymal transition (EMT) is a major component of peritoneal injury associated with peritoneal fibrosis in the end stage of renal disease; however, at present, the underlying mechanisms remain unclear. Thus, in the present study, uric acid (UA)-induced EMT of peritoneal mesothelial cells was investigated by western-blot and immunofluorescence staining. The results revealed that peritoneal mesothelial cells stimulated with UA underwent EMT, as demonstrated by the decreased expression of epithelial markers (E-cadherin) and an increased expression of mesenchymal markers $(\alpha$-smooth muscle actin and vimentin). Additionally, it was reported that UA could facilitate the progression of EMT of peritoneal mesothelial cells via EMT transcription pathways, including transforming growth factor- $\beta 1 /$ mothers against decapentaplegic homolog 3 and P38/mitogen-activated protein kinase by western-blot and reverse transcription semi-quantitative polymerase chain reaction. The results of the present study suggest that UA could promote EMT and may contribute to peritoneal chronic disease. Furthermore, the data obtained suggest that the levels of blood UA may account for the development of EMT; thus, lowering the levels of blood UA may be beneficial to inhibit the occurrence and development of peritoneal fibrosis.
\end{abstract}

\section{Introduction}

End-stage renal disease (ESRD) is generally diagnosed when the glomerular filtration rate (GFR) is $<15 \mathrm{ml} / \mathrm{min} / 1.73 \mathrm{~m}^{2}$,

Correspondence to: Professor Yan Lin, Department of Endocrine and Metabolic Diseases, Shaanxi Provincial People's Hospital, 256 Youyixi Road, Xi'an, Shaanxi 710068, P.R. China

E-mail: duanzhaoyang2005@126.com

Key words: uric acid, epithelial-to-mesenchymal transition, TGF- $\beta 1 /$ Smad3, P38 which can be similar to that in uremia $(1,2)$. ESRD patients have two treatment options: Long-term dialysis or kidney transplantation. Owing to problems arising from donors, matching type and race, numerous patients have to undergo dialysis treatments, including hemodialysis (HD) or peritoneal dialysis (PD), as an alternative to renal replacement therapy (3). Compared with HD, PD has been associated with notably better or at least similar survival of patients, lower cost and is superior to retaining residual renal function $(4,5)$; however, few patients are reluctant to utilize PD for extended periods due to the progression of peritoneal fibrosis. Thus, applying $\mathrm{PD}$ for $>10$ years is exceedingly rare (6). The occurrence of peritoneal fibrosis is mainly due to the composition of PD solution (high-glucose content, low $\mathrm{pH}$, elevated osmolality, increased concentration of lactate and the degradation products of glucose), uremic toxins, refractory or recurrent infectious peritonitis, and chronic inflammation (7-9). Therefore, understanding the origin of peritoneal fibrosis is critical to maintaining the integrity of the peritoneal membrane and to prolong PD treatment in such patients. At present, no studies have investigated this; thus, the underlying mechanism is unclear.

Uric acid (UA) is the metabolic end product of nucleic acid purine (including nucleic acids in food), which is mainly eliminated via renal excretion. The levels of UA are an indispensable marker in the detection of rare inborn errors of metabolism of the degradation system of purine nucleotides (10). Hyperuricemia has been frequently observed in patients with chronic kidney disease (CKD) due to decreases in GFR. In numerous, serum UA has been reported as a predictor of the progression of renal disease, as $>90 \%$ of hyperuricemia is due to the impaired renal excretion of UA $(11,12)$. Thus, whether high levels of blood UA have an effect on peritoneal fibrosis, as well as reducing the levels of UA alleviates peritoneal fibrosis, requires further investigation. On this basis, the present study employed peritoneal mesothelial cells to determine the role of serum UA.

\section{Materials and methods}

Reagents. Fetal bovine serum(FBS; 10\%), Dulbecco's Modified Eagle's medium (DMEM)/F12 medium, 1\% (v/v) penicillin and streptomycin were obtained from Invitrogen (Thermo 
Fisher Scientific, Inc., Waltham, MA, USA). UA, Triton X-100, MTT, dimethyl sulfoxide (DMSO), DAPI, Hoechst 33342 were purchased from Sigma-Aldrich (Merck KGaA, Darmstadt, Germany). Anti-phosphorylated (p)-P38, anti-total (t)-P38, anti-mothers against decapentaplegic homolog 3 (Smad3), anti-p-Smad3, anti-E-cadherin, anti-transforming growth factor (TGF)- $\beta 1$, anti- $\alpha$-smooth muscle actin (SMA), anti-vimentin and anti- $\beta$-actin were purchased from Santa Cruz Biotechnology, Inc. (Dallas, TX, USA). An enhanced chemiluminescence kit was also purchased (Pierce; Thermo Fisher Scientific, Inc.).

Cell culture. The cell line employed in the present study was the human peritoneal mesothelial cell line, HMrSV5, which was obtained from the Cell Culture Centre (Chinese Academy of Medical Sciences, Beijing, China). HMrSV5 cells were cultured in DMEM/F12 medium containing $10 \% \mathrm{FBS}$ at $37^{\circ} \mathrm{C}$ in a humidified environment with $5 \% \mathrm{CO}_{2}$. The cells were dissociated using $0.2 \%$ trypsin plus $0.02 \%$ EDTA solution at $37^{\circ} \mathrm{C}$ and sub-cultured once in 4 days. The cells were then rinsed with $\mathrm{PBS}$, the medium was exchanged and the cells were cultured further.

MTT assay. Cell viability was determined by an MTT assay. Briefly, HMrSV5 cells in the logarithmic growth phase were cultured at a density of $5 \times 10^{3}$ cells/well in 96 -well plates. The cells were exposed to various concentrations $(0,200,400,600$, $800,1,000,1,200$ and $1,500 \mu \mathrm{mol} / \mathrm{l})$ of UA for 24,48 and $72 \mathrm{~h}$. For analysis, $20 \mu \mathrm{l}$ of MTT substrate was added to each well and the plates were incubated for an additional $4 \mathrm{~h}$ at $37^{\circ} \mathrm{C}$ with $5 \% \mathrm{CO}_{2}$. The medium was removed, and the cells were solubilized in $150 \mu \mathrm{l}$ DMSO to dissolve the colored formazan crystals for $15 \mathrm{~min}$. The absorbance of each well was recorded on a microplate spectrophotometer at $490 \mathrm{~nm}$ using a microplate reader (ASYS Expert Plus, ASYS Hitech GmbH, Eugendorf, Austria). Relative cellular growth was determined using the ratio of average absorbance in treatment cells vs. the average absorbance in control cells. The cell viability was calculated as the ratio of optical densities. UA concentrations of 200 and $400 \mu \mathrm{mol} / 1$ were used in the experiments below.

Terminal deoxynucleotidyl-transferase-mediated dUTP nick end labelling (TUNEL) assay. TUNEL assays were performed using an ApopTag ${ }^{\circledR}$ kit (Merck KGaA) according to the manufacturer's protocols. HMrSV5 cells were fixed with $4 \%$ paraformaldehyde solution for $30 \mathrm{~min}$ and then blocked using PBS buffer containing $2 \% \mathrm{H}_{2} \mathrm{O}_{2}$ for $5 \mathrm{~min}$ at room temperature. Following washing with PBS, cells were treated with $0.1 \%$ Triton X-100 on ice for $2 \mathrm{~min}$ to increase the permeability of the cell membrane. Subsequently, the cells were incubated with $50 \mu \mathrm{l}$ TUNEL reagent in a humidified chamber for $1 \mathrm{~h}$ at $37^{\circ} \mathrm{C}$. To detect the nuclei, the cells were incubated with DAPI for $2 \mathrm{~min}$ at room temperature in the dark and mounted in neutral resin. An epifluorescent microscope (FV300, Olympus Corporation, Tokyo, Japan) was used to detect apoptotic cells (magnification, x40). A total of 3 randomly selected number of fields were examined.

Immunofluorescence staining. HMrSV5 cells were seeded in 6-well plates. After the indicated treatment, the cells were fixed with $4 \%$ paraformaldehyde for $15 \mathrm{~min}$ at $0^{\circ} \mathrm{C}$, permeabilized with $0.2 \%$ Triton X-100 in PBS for $5 \mathrm{~min}$, and blocked with $50 \mathrm{mg} / \mathrm{ml}$ bovine serum albumin (Sigma-Aldrich) in PBS at room temperature for $30 \mathrm{~min}$. Following incubation with primary antibodies against vimentin (1:200; sc-5565; Santa Cruz Biotechnology, Inc.) and E-cadherin (1:200; sc-7870; Santa Cruz Biotechnology, Inc.) overnight at $4^{\circ} \mathrm{C}$, the cells were washed three times with PBS. The tetramethylrhodamine-conjugated goat anti-mouse IgG antibody (1:100; sc-57606 Santa Cruz Biotechnology, Inc.) was applied for $0.5 \mathrm{~h}$ at room temperature. The cell nuclei were counterstained with Hoechst 33342 for $15 \mathrm{~min}$ at room temperature and the images were captured using a fluorescence microscope (magnification, x10; excitation wavelength, $430 \mathrm{~nm}$; FV300, Olympus Corporation).

Western blot analysis. HMrSV5 cells were lysed with radioimmunoprecipitation assay lysis buffer (Beyotime Institute of Biotechnology, Haimen, China) on ice for $30 \mathrm{~min}$ and centrifuged at $15,000 \mathrm{x} \mathrm{g}$ at $4^{\circ} \mathrm{C}$ for $30 \mathrm{~min}$. The supernatant was collected, and protein concentrations were determined using a bicinchoninic acid assay kit (Pierce; Thermo Fisher Scientific, Inc.). Equal protein lysate samples $(10 \mu \mathrm{g})$ were separated via $12 \%$ SDS-PAGE and then transferred to nitrocellulose membranes. The membranes were blocked at room temperature for $2 \mathrm{~h}$ in TBS with $0.1 \%$ Tween-20 (TBS-T) containing $5 \%$ skim milk, followed by incubation with primary antibodies at $4^{\circ} \mathrm{C}$ overnight. The primary antibodies used in the present study were: Anti-p-P38 (1:400; sc-7973), anti-P38 (1:400; sc-7972), anti-Smad3 (1:400; sc-101154), anti-p-Smad3 (1:400; sc-517575), anti-E-cadherin (1:400; sc-7870), anti-TGF- $\beta 1$ (1:400; sc-146), anti- $\alpha$-SMA (1:400; sc-324317), anti-vimentin $(1: 400 ;$ sc-5565) and anti- $\beta$-actin $(1: 400 ;$ sc-47778). The membrane was then incubated with horseradish peroxidase-conjugated secondary antibodies (sc-2039 and sc-2094; Santa Cruz Biotechnology, Inc.) for $2 \mathrm{~h}$ at room temperature. Blots were developed using an enhanced chemiluminescence kit (Pierce; Thermo Fisher Scientific, Inc.). Relative expression was quantified using Quantity One Software (version 643274, rev. A; Bio-Rad Laboratories, Inc., Hercules, CA, USA).

\section{Reverse transcription semi-quantitative polymerase chain} reaction $(R T-q P C R)$. Total RNA was extracted from HMrSV5 cells using TRIzol ${ }^{\circledR}$ RNA isolation (Gibco; Thermo Fisher Scientific, Inc.) and purified with DNase I (Invitrogen; Thermo Fisher Scientific, Inc.). RNA (1 $\mu \mathrm{g})$ was reverse transcribed into the first strand of cDNA using a SuperScript Reverse Transcriptase system (Invitrogen; Thermo Fisher Scientific, Inc.) according to the manufacturer's protocols. qPCR reactions were performed using a SuperScript ${ }^{\mathrm{TM}}$ First-Strand Synthesis kit (Invitrogen; Thermo Fisher Scientific, Inc.) and a Gene Amp PCR system 9700 (PerkinElmer, Inc., Waltham, MA, USA). qPCR was performed with the following thermocycling conditions: First at $95^{\circ} \mathrm{C}$ for $3 \mathrm{~min}, 30$ cycles at $95^{\circ} \mathrm{C}$ for $30 \mathrm{sec}$, with different annealing temperatures $\left(55^{\circ} \mathrm{C}\right.$ for TGF- $\beta 1 ; 60^{\circ} \mathrm{C}$ for GAPDH) for $30 \mathrm{sec}$, at $72^{\circ} \mathrm{C}$ for $30 \mathrm{sec}$, and extension at $72^{\circ} \mathrm{C}$ for $10 \mathrm{~min}$. The amplified products were separated by electrophoresis on a $2 \%$ agarose gel and visualized by ethidium bromide staining for $30 \mathrm{~min}$ at room temperature. Each product was visualized after separation and GAPDH was used 
A
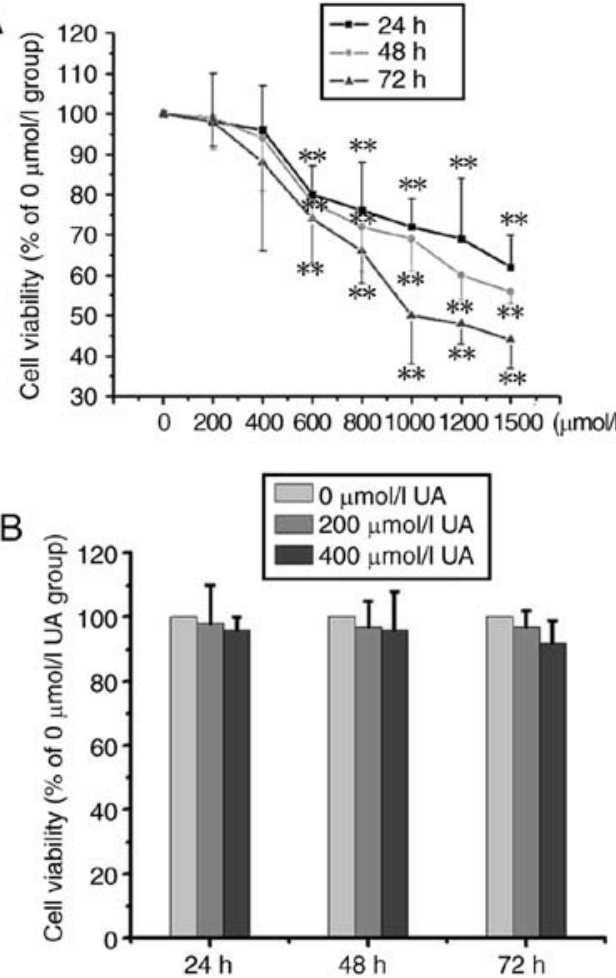

C

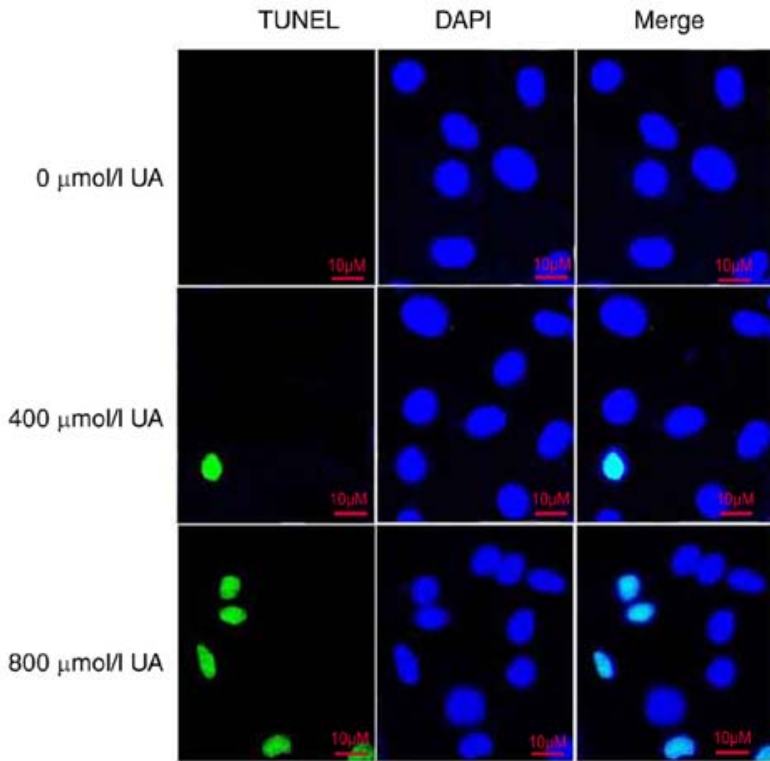

D

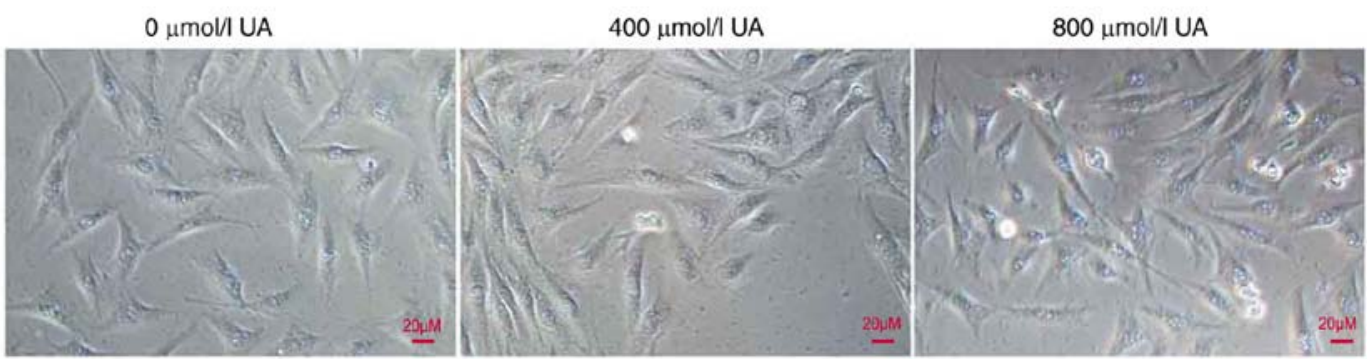

Figure 1. UA inhibits cell viability in peritoneal mesothelial cells. (A) HMrSV5 cells were treated with $0-1,500 \mu$ mol/1 of UA for 24,48 and $72 \mathrm{~h}$, and the cell viability was detected via an MTT assay $(\mathrm{n}=3)$. Values are presented as the mean \pm standard deviation; ${ }^{* *} \mathrm{P}<0.01$ vs. $0 \mu$ mol/1 group. $(\mathrm{B}) \mathrm{Cells}$ were treated with 0,200 and $400 \mu \mathrm{mol} / 1$ of UA for 24,48 and $72 \mathrm{~h}$, and cell viability was detected using an MTT assay. (C) Cells were treated with 0,400 and $800 \mu \mathrm{mol} / 1$ of UA for $48 \mathrm{~h}$, and the TUNEL-stained cells were detected. Scale bar $=10 \mu \mathrm{m}$. (D) Cells were treated as aforementioned, and the cells were observed under a light microscope. Scale bar, $20 \mu \mathrm{m}$. Results are presented as the mean \pm standard deviation. TUNEL, terminal deoxynucleotidyl-transferase-mediated dUTP nick end labelling; UA, uric acid.

to normalize the mRNA expression levels. Image density was quantified with a FluoroImager SI (GE Healthcare, Chicago, IL, USA). The sequences of TGF- $\beta 1$ and GAPDH genes were obtained from the GenBank database (https://www.ncbi.nlm. nih.gov/genbank/), and specific primers were designed over an exon-exon junction with Primer Premier 5.0 (Premier Biosoft International, Palo Alto, CA, USA).

The primers used for amplification of TGF- $\beta 1$ were as follows: Forward, 5'-AACATGATCGTGCGCTCTGCAAGT GCAGC-3' and reverse, 5'-AAGGAATAGTGCAGACAG GCAGGA-3'; GAPDH, forward, 5'-CCTTCATTGACCTCA ACTAC-3' and reverse, 5'-CCAAAGTTGTCATGGATG-3'.

Statistical analysis. Statistical analysis was performed using SPSS 19.0 (IBM Corp., Armonk, NY, USA). All values were expressed as the mean \pm standard deviation, which were obtained from at least three independent experiments for each condition. Statistical significance was determined by performing one-way analyses of variance followed by Tukey's multiple comparison test. $\mathrm{P}<0.05$ was considered to indicate a statistically significant difference, and $\mathrm{P}<0.01$ was considered to indicate a highly statistically significant difference.

\section{Results}

Effects of UA on the cell viability of peritoneal mesothelial cells. In order to examine the cytotoxic effects of UA on peritoneal mesothelial cells, HMrSV5 cells were incubated with UA at various concentrations $(0-1,500 \mu \mathrm{mol} / \mathrm{l})$ for 24-72 h, and the cell viability was determined using an MTT assay. As presented in Fig. 1A, UA-treated HMrSV5 cells $(\leq 400 \mu \mathrm{mol} / \mathrm{l})$ did not exhibit significant reductions in cell viability compared with the control $(\mathrm{P}>0.05)$; however, above this concentration, the cell viability significantly decreased $(\mathrm{P}<0.01)$. In particular, UA of 200 or $400 \mu \mathrm{mol} / 1$ did not notably alter the cell viability at different durations as demonstrated in Fig. 1B. To further analyze UA-induced apoptosis, a TUNEL experiment was conducted. The results 
A
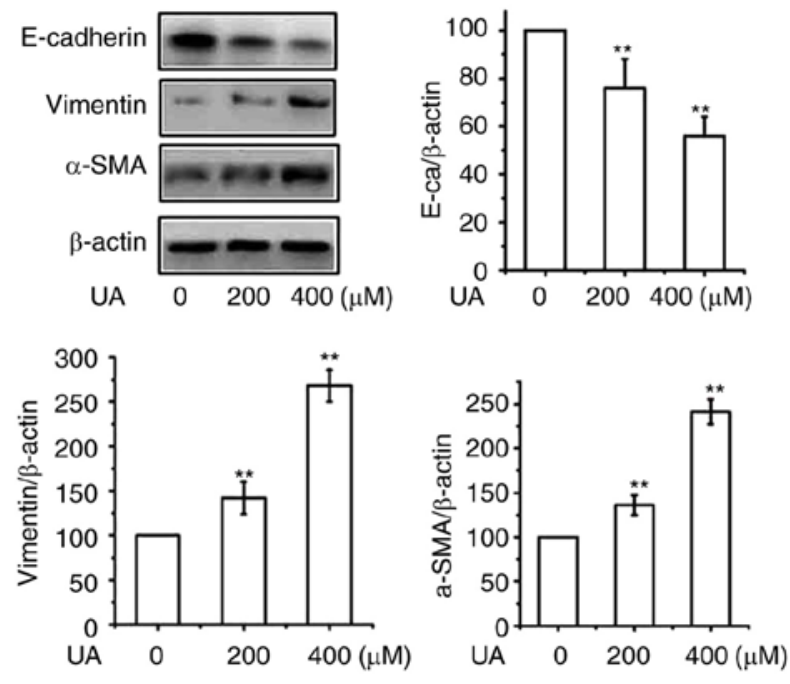

C
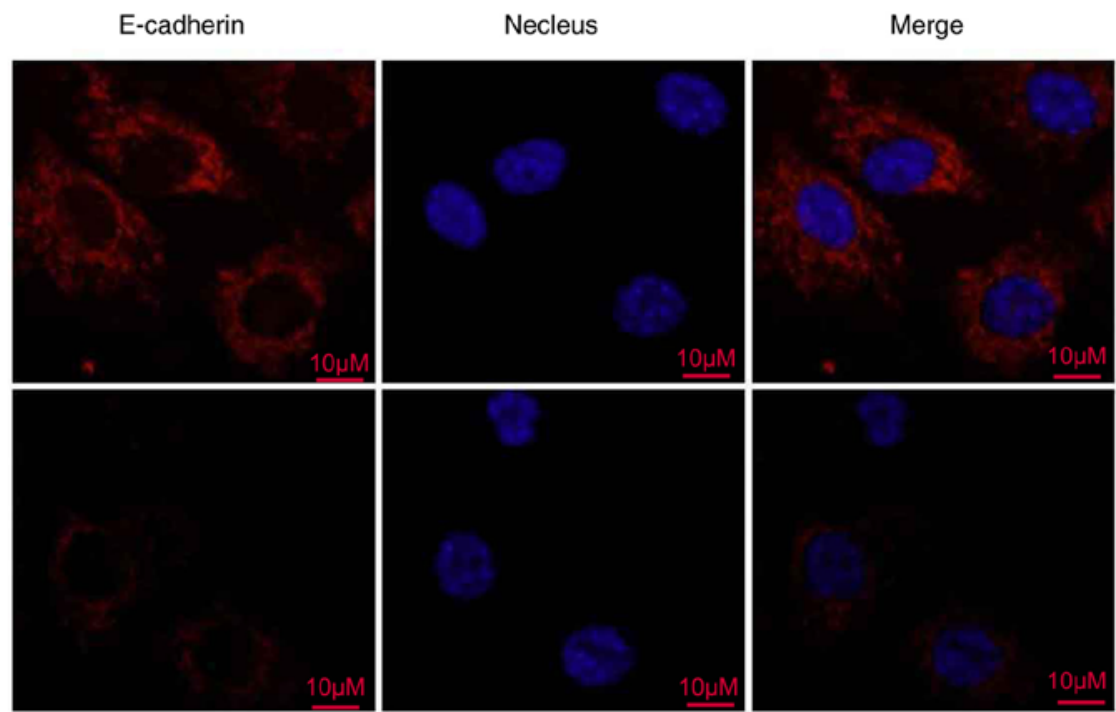

Figure 2. UA promotes epithelial-mesenchymal transformation in peritoneal mesothelial cells. (A) HMrSV5 cells were treated with 0,200 and $400 \mu$ mol/1 of UA for $48 \mathrm{~h}$. Cell lysates were subjected to immunoblot analysis using antibodies against E-cadherin, vimentin, $\alpha$-SMA or $\beta$-actin. Values are presented as the mean \pm standard deviation of at least three independent experiments. $\beta$-actin was used as a loading control $(* * \mathrm{P}<0.01$ vs. control). (B) Cells were treated with 0 or $400 \mu \mathrm{mol} / 1 \mathrm{UA}$ for $48 \mathrm{~h}$, and vimentin was detected via immunofluorescence. Scale bar, $10 \mu \mathrm{m}$. (C) Cells were treated as aforementioned, and the expression of E-cadherin was detected via immunofluorescence. Scale bar=10 $\mu \mathrm{m}$. $\alpha$-SMA, $\alpha$-smooth muscle actin; UA, uric acid.

revealed that $\leq 400 \mu \mathrm{mol} / 1 \mathrm{UA}$ is almost non-toxic to cells, whereas $>400 \mu \mathrm{mol} / 1$ of UA was observed to increase the cytotoxicity of the cells (Fig. 1C). Similar results were acquired via analysis under a light microscope (Fig. 1D). Following treatment with $0-400 \mu \mathrm{mol} / 1 \mathrm{UA}$, apoptosis was not notably induced. In the follow-up epithelial-mesenchymal transition (EMT) experiments, UA concentrations of 200 and $400 \mu \mathrm{mol} / 1$ were used.

Effects of UA on EMT of peritoneal mesothelial cells. A previous study demonstrated that EMT contributes to the development of peritoneal fibrosis (13). In order to understand whether UA is involved in the regulation of peritoneal fibrosis, the effects of UA on the EMT of cultured human peritoneal mesothelial cells were examined. As demonstrated in Fig. 2A, compared with the control, treatment with UA significantly upregulated the expression of $\alpha$-SMA and vimentin, and significantly downregulated the expression of E-cadherin in a dose-dependent manner, following treatment with 0,200 and $400 \mu \mathrm{mol} / 1 \mathrm{UA}$ for $48 \mathrm{~h}$. The effects of UA-induced EMT of HMrSV5 cells were subsequently assessed via immunofluorescence. As presented in Fig. 2B and C, it was observed that treatment with UA led to the increased the expression of vimentin and decreased E-cadherin expression, indicating the characteristics of a mesenchymal phenotype. These results confirm that UA acts positively to regulate EMT in peritoneal mesothelial cells.

Effects of UA on TGF- $\beta 1 / S m a d 3$ pathway of peritoneal mesothelial cells. It is well known that activation of TGF- $\beta 1 / \mathrm{Smad} 3$ signaling is critical for the development of EMT (14). Thus, whether UA may regulate EMT via the activation of the signaling pathway in HMrSV5 cells was examined. To demonstrate whether UA serves a role in regulating the activation of the TGF- $\beta 1 / \mathrm{Smad} 3$ pathway during EMT, the expression levels of TGF- $\beta 1$ and Smad3 were determined. As demonstrated 

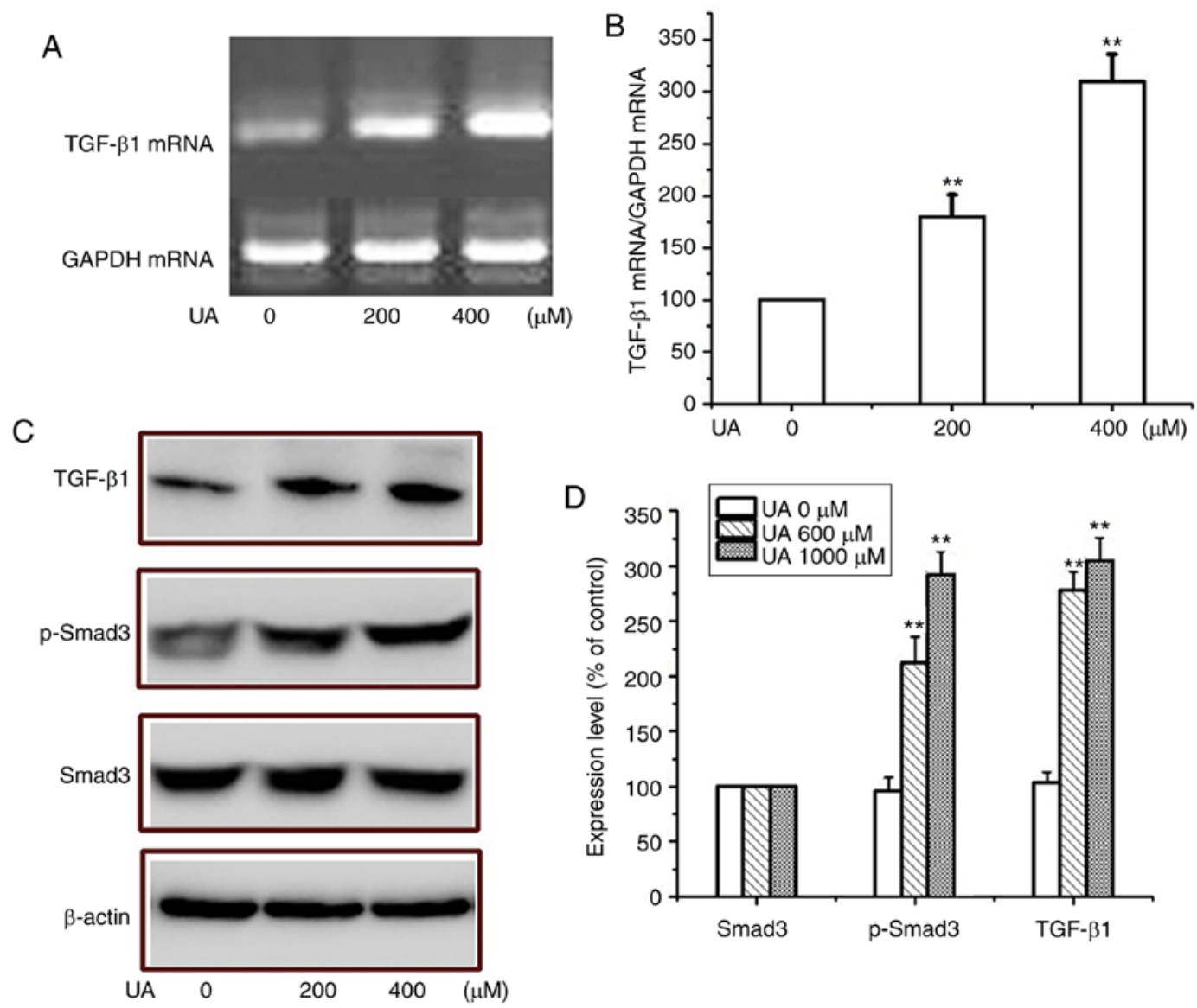

Figure 3. UA activates the TGF- $\beta /$ Smad signaling pathway in peritoneal mesothelial cells. (A and B) HMrSV5 cells were treated with 0,200 and $400 \mu$ mol/1 of UA for $48 \mathrm{~h}$, and the expression of TGF- $\beta 1$ was detected via reverse transcription semi-quantitative polymerase chain reaction. GAPDH served as a loading control. Values are presented as the mean \pm standard deviation of at least three independent experiments $(" \mathrm{*} \times 0.01$ vs. control). (C and D) Cells were treated as aforementioned, and cell lysates were subjected to immunoblot analysis with antibodies against TGF- $\beta 1$, Smad3, $\mathrm{p}$-Smad 3 or $\beta$-actin. $\beta$-actin was used as a loading control ( ${ }^{* *} \mathrm{P}<0.01$ vs. control). p, phosphorylated; Smad, mothers against decapentaplegic; TGF- $\beta 1$, transforming growth factor $\beta 1$; UA, uric acid.

in Fig. 3A and B, following UA treatment, the expression of TGF- $\beta 1$ was significantly increased compared with the control group, which may be associated with upregulation of $\alpha$-SMA expression and downregulation of E-cadherin. Similarly, as presented in Fig. 3C and D, UA treatment promoted the phosphorylation of Smad3 in a dose-dependent manner; however, the levels of total Smad3 did not change significantly. Therefore, UA may serve a critical role in mediating the activation of TGF- $\beta 1 / \mathrm{Smad} 3$ in peritoneal mesothelial cells undergoing EMT.

Effects of UA on mitogen-activated protein kinase $(M A P K) / P 38$ pathway of peritoneal mesothelial cells. P38 MAPKs serve important roles in the invasion and fibrosis of tumor cells. It has been suggested that P38 MAPK signaling regulates EMT (15). Thus, the effects of UA-induced EMT were determined by investigating the important pathway of MAPK-p38 proteins in HMrSV5 cells. As demonstrated in Fig. 4A and B, the exposure of peritoneal mesothelial cells to UA promoted the expression of $\mathrm{p}-\mathrm{P} 38$, which indicated that UA treatment activated the pathway in a dose-dependent manner; however, the expression of t-P38 did not change significantly. These findings suggest that UA treatment markedly induced EMT and regulated MAPK-P38 signaling pathway in HMrSV5 cells.
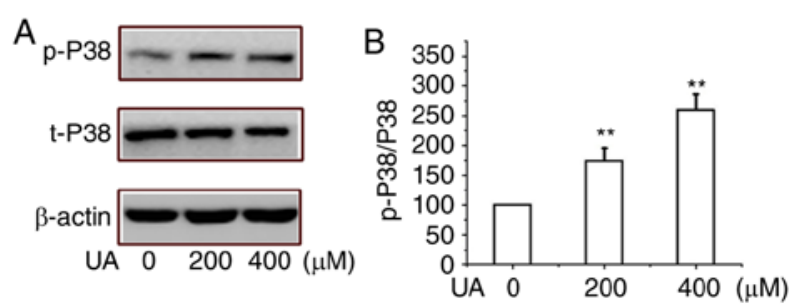

Figure 4. UA activates P38 signaling pathway in peritoneal mesothelial cells. (A and B) HMrSV5 cells were treated with 0,200 and $400 \mu \mathrm{mol} / 1$ of UA for $48 \mathrm{~h}$. Cell lysates were subjected to immunoblot analysis with antibodies against t-P38, p-P38 or $\beta$-actin. Values are presented as the mean \pm standard deviation of at least three independent experiments. $\beta$-actin was used as loading control. ${ }^{* *} \mathrm{P}<0.01$ vs. Control. p, phosphorylated; t, total; UA, uric acid.

\section{Discussion}

Recent studies have demonstrated that EMT of peritoneal epithelial cells may be one of the mechanisms underlying peritoneal fibrosis, in which epithelial cells lose their characteristic cell-cell junctions, polarized cell-surface molecules and apical-basolateral polarity, while acquiring properties typical of mesenchymal cells and increased migratory, invasive, and fibrogenic potentials $(16,17)$. UA has been reported to serve a pathogenic role in CKD; however, its 
effect on EMT or peritoneal fibrosis has not yet been elucidated. Therefore, in the present study, the effects of UA on the EMT of peritoneal mesothelial cells were investigated. It was demonstrated that UA induced downregulation of E-cadherin, and upregulation of $\alpha$-SMA and vimentin in human peritoneal mesothelial cells in vitro. Overall, it was proposed that UA promotes the EMT process of peritoneal mesothelial cells. Previous studies have reported that oxidative stress serves an important role in the development of fibrosis under various pathological conditions, while few studies suggest that UA may induce oxidative stress, and be a mediator of EMT $(18,19)$. UA may indirectly promote the development of EMT by inducing oxidative stress. Conversely, UA induces the release of cellular inflammatory mediators, including monocyte chemoattractant protein-1, cyclooxygenase- 2 and tumor necrosis factor- $\alpha$ (20). Notably, it is well established that these pro-inflammatory cytokines are strongly associated with the occurrence of EMT $(21,22)$.

Furthermore, in the present study, signaling cascades involved in the effects of UA-induced EMT were investigated. Emerging evidence has demonstrated that TGF- $\beta 1 / \mathrm{Smad} 3$ signaling serves a pivotal role in EMT (23). The results also indicated that TGF- $\beta 1$ is crucial for the initiation of EMT, and the TGF- $\beta 1 / \mathrm{Smad} 3$ pathway is regulated by UA, which can directly promote EMT. Numerous studies have previously demonstrated that the activity of the MAPK pathway is required for TGF- $\beta 1$-induced EMT in mammary epithelial cells $(24,25)$. The present study reported that exposure of peritoneal mesothelial cells to UA promoted the expression of p-P38, which suggested that UA-induced EMT is closely associated with the activation of $\mathrm{P} 38$.

In conclusion, the present study investigated whether UA promotes TGF- $\beta 1$-induced EMT, as well as the potential pathways involved in this crucial transition of peritoneal mesothelial cells. The findings demonstrate a potential association between UA and the progression of EMT in peritoneal mesothelial cells. Future investigation is required to further understand the role of UA in peritoneal fibrosis during long-term peritoneal dialysis. The potential for alleviating peritoneal fibrosis by lowering the serum UA levels is promising, and thus should be evaluated in well-designed randomized controlled studies in the future.

\section{Acknowledgements}

Not applicable.

\section{Funding}

No funding was received.

\section{Availability of data and materials}

All data generated or analyzed during this study are included in this published article.

\section{Authors' contributions}

C-YD was the guarantor of integrity for the entire study and responsible for the study concepts, the definition of intellectual content, the clinical studies, and statistical analysis. YL was responsible for the study concepts, the study design, literature research, statistical analysis and manuscript preparation, editing and review. $\mathrm{JH}$ was responsible for aquisition, analysis and interpretation of data, the definition of intellectual content and manuscript preparation, editing and review. KW performed experimental studies and data analysis. C-YZ performed data acquisition. All authors read and approved the final manuscript.

\section{Ethics approval and consent to participate}

Not applicable.

\section{Patient consent for publication}

Not applicable.

\section{Competing interests}

The authors declare that they have no competing interests.

\section{References}

1. Grams ME, Li L, Greene TH, Tin A, Sang Y, Kao WH, Lipkowitz MS, Wright JT, Chang AR, Astor BC and Appel LJ: Estimating time to ESRD using kidney failure risk equations: Results from the African American study of kidney disease and hypertension (AASK). Am J Kidney Dis 65: 394-402, 2015.

2. JunM,TurinTC,WoodwardM,Perkovic V,Lambers HeerspinkHJ, Manns BJ, Tonelli M and Hemmelgarn BR: Assessing the validity of surrogate outcomes for ESRD: A meta-analysis. J Am Soc Nephrol 26: 2289-2302, 2015.

3. Purnell TS, Luo X, Cooper LA, Massie AB, Kucirka LM, Henderson ML, Gordon EJ, Crews DC, Boulware LE and Segev DL: Association of race and ethnicity with live donor kidney transplantation in the United States from 1995 to 2014. JAMA 319: 49-61, 2018.

4. Teixeira JP, Combs SA and Teitelbaum I: Peritoneal dialysis: Update on patient survival. Clin Nephrol 83: 1-10, 2015.

5. Weinhandl ED, Foley RN, Gilbertson DT, Arneson TJ, Snyder JJ and Collins AJ: Propensity-matched mortality comparison of incident hemodialysis and peritoneal dialysis patients. J Am Soc Nephrol 21: 499-506, 2010.

6. Morinelli TA, Luttrell LM, Strungs EG and Ullian ME: Angiotensin II receptors and peritoneal dialysis-induced peritoneal fibrosis. Int J Biochem Cell Biol 77: 240-250, 2016.

7. Fernández-Perpén A, Pérez-Lozano ML, Bajo MA Albar-Vizcaino P, Sandoval Correa P, del Peso G, Castro MJ, Aguilera A, Ossorio M, Peter ME, et al: Influence of bicarbonate/low-GDP peritoneal dialysis fluid (BicaVera) on in vitro and ex vivo epithelial-to-mesenchymal transition of mesothelial cells. Perit Dial Int 32: 292-304, 2012.

8. Ueno T, Nakashima A, Doi S, Kawamoto T, Honda K, Yokoyama Y, Doi T, Higashi Y, Yorioka N, Kato Y, et al: Mesenchymal stem cells ameliorate experimental peritoneal fibrosis by suppressing inflammation and inhibiting TGF- $\beta 1$ signaling. Kidney Int 84: 297-307, 2013.

9. Huh JY, Seo EY, Lee HB and Ha H: Glucose-based peritoneal dialysis solution suppresses adiponectin synthesis through oxidative stress in an experimental model of peritoneal dialysis. Perit Dial Int 32: 20-28, 2012.

10. Jasinge E, Kularatnam GAM, Dilanthi HW, Vidanapathirana DM, Jayasena KLSPKM, Chandrasiri NDPD, Indika NLR, Ratnayake PD, Gunasekara VN, Fairbanks LD and Stiburkova B: Uric acid, an important screening tool to detect inborn errors of metabolism: A case series. BMC Res Notes 10: 454, 2017.

11. Anker SD, Doehner W, Rauchhaus M, Sharma R, Francis D, Knosalla C, Davos CH, Cicoira M, Shamim W, Kemp M, et al: Uric acid and survival in chronic heart failure: Validation and application in metabolic, functional, and hemodynamic staging. Circulation 107: 1991-1997, 2003. 
12. Richette $P$ and Bardin T: Gout. Lancet 375: 318-328, 2010.

13. Wakabayashi K, Hamada C, Kanda R, Nakano T, Io $H$, Horikoshi S and Tomino Y: Adipose-derived mesenchymal stem cells transplantation facilitate experimental peritoneal fibrosis repair by suppressing epithelial-mesenchymal transition. J Nephrol 27: 507-514, 2014.

14. Brodeur AC, Roberts-Pilgrim AM, Thompson KL, Franklin CL and Phillips CL: Transforming growth factor- $31 / \mathrm{Smad} 3$-independent epithelial-mesenchymal transition in type I collagen glomerulopathy. Int J Nephrol Renovasc Dis 10: 251-259, 2017.

15. Hipp S, Berg D, Ergin B, Schuster T, Hapfelmeier A, Walch A Avril S, Schmalfeldt B, Höfler H and Becker KF: Interaction of Snail and p38 mitogen-activated protein kinase results in shorter overall survival of ovarian cancer patients. Virchows Arch 457: 705-713, 2010.

16. Ouanouki A, Lamy S and Annabi B: Periostin, a signal transduction intermediate in TGF- $\beta$-induced EMT in U-87MG human glioblastoma cells, and its inhibition by anthocyanidins. Oncotarget 9: 22023-22037, 2018.

17. Tennakoon AH,Izawa T, Kuwamura M and Yamate J: Pathogenesis of type 2 epithelial to mesenchymal transition (EMT) in renal and hepatic fibrosis. J Clin Med 5: E4, 2015.

18. Liu RM and Gaston Pravia KA: Oxidative stress and glutathione in TGF-beta-mediated fibrogenesis. Free Radic Biol Med 48: $1-15,2010$
19. Zharikov S, Krotova K, Hu H, Baylis C, Johnson RJ, Block ER and Patel J: Uric acid decreases NO production and increases arginase activity in cultured pulmonary artery endothelial cells. Am J Physiol Cell Physiol 295: C1183-C1190, 2008.

20. Kang DH, Nakagawa T, Feng L, Watanabe S, Han L, Mazzali M, Truong L, Harris R and Johnson RJ: A role for uric acid in the progression of renal disease. J Am Soc Nephrol 13: 2888-2897, 2002.

21. Strippoli R, Moreno-Vicente R, Battistelli C, Cicchini C, Noce V, Amicone L, Marchetti A, Del Pozo MA and Tripodi M: Molecular mechanisms underlying peritoneal EMT and fibrosis. Stem Cells Int 2016: 3543678, 2016.

22. Li S, Lu J, Chen Y, Xiong N, Li L, Zhang J, Yang H, Wu C, Zeng $\mathrm{H}$ and Liu Y: MCP-1-induced ERK/GSK-3 $\beta /$ Snail signaling facilitates the epithelial-mesenchymal transition and promotes the migration of MCF-7 human breast carcinoma cells. Cell Mol Immunol 14: 621-630, 2017.

23. Margetts PJ, Bonniaud P, Liu L, Hoff CM, Holmes CJ, West-Mays JA and Kelly MM: Transient overexpression of TGF- $\{$ beta 1 induces epithelial mesenchymal transition in the rodent peritoneum. J Am Soc Nephrol 16: 425-436, 2005.

24. Mulder KM: Role of Ras and Mapks in TGFbeta signaling. Cytokine Growth Factor Rev 11: 23-35, 2000.

25. Witte D, Otterbein H, Förster M, Giehl K, Zeiser R, Lehnert H and Ungefroren $\mathrm{H}$ : Negative regulation of TGF- $\beta 1$-induced MKK6-p38 and MEK-ERK signalling and epithelial-mesenchymal transition by Rac1b. Sci Rep 7: 17313, 2017. 